\title{
In Vivo Imaging Reveals Dissociation between Caspase Activation and Acute Neuronal Death in Tangle-Bearing Neurons
}

\author{
Tara L. Spires-Jones, ${ }^{1}$ Alix de Calignon, ${ }^{1}$ Toshifumi Matsui, ${ }^{1}$ Cindy Zehr, ${ }^{3}$ Rose Pitstick,,${ }^{4}$ Hai-Yan Wu, ${ }^{1}$ \\ Jennifer D. Osetek, ${ }^{1}$ Phillip B. Jones, ${ }^{1,2}$ Brian J. Bacskai, ${ }^{1}$ Mel B. Feany, ${ }^{5}$ George A. Carlson, ${ }^{4}$ Karen H. Ashe, ${ }^{6}$ Jada Lewis, ${ }^{3}$ \\ Bradley T. Hyman ${ }^{1}$ \\ ${ }^{1}$ MassGeneral Institute for Neurodegenerative Disease and ${ }^{2}$ Martinos Center for Biomedical Imaging, Massachusetts General Hospital, Harvard Medical \\ School, Charlestown, Massachusetts 02129, ${ }^{3}$ Neurogenetics, Neurotransgenics and Neuropathology Laboratories, Mayo Clinic, Jacksonville, Florida 32224, \\ ${ }^{4}$ McLaughlin Research Institute, Great Falls, Montana 59405, 5 Department of Pathology, Brigham and Women's Hospital and Harvard Medical School, \\ Boston, Massachusetts 02115, and ' $D$ Department of Neurology, University of Minnesota Medical School, Minneapolis, Minnesota 55455
}

Accumulation of neurofibrillary tangles (NFTs) in Alzheimer's disease correlates with neuronal loss and cognitive decline, but the precise relationship between NFTs and neuronal death and downstream mechanisms of cell death remain unclear. Caspase cleaved products accumulate in tangles, implying that tangles may contribute to apoptotic neuronal death. To test this hypothesis, we developed methods using multiphoton imaging to detect both neurofibrillary pathology and caspase activation in the living mouse brain. We examined $r T g 4510$ mice, a reversible mouse model of tauopathy that develops tangles and neuronal loss. Only a small percentage of imaged neurons were caspase activity positive, but the vast majority of the cells with active caspases contained NFTs. We next tested the hypothesis that caspase activation led to acute, apoptotic neuronal death. Caspase positive cell bodies did not degenerate over hours of imaging, despite the presence of activated executioner caspases. Suppression of the transgene, which stops ongoing death, did not suppress caspase activity. Finally, histochemical assessments revealed evidence of caspase-cleaved tau, but no TUNEL (terminal deoxynucleotidyl transferase-mediated biotinylated UTP nick end labeling) positive or apoptotic nuclei. With the novel technique of observing NFTs and caspase activation in the living brain, we demonstrate that aggregated tau in neurons can be associated with caspase activation, but that caspase activation is not sufficient to cause acute neuronal death in this model.

Key words: Alzheimer's; neurofibrillary tangle; caspase; apoptosis; multiphoton imaging; tauopathy

\section{Introduction}

Conventional histological studies of human Alzheimer's disease (AD) and animal models of tauopathies have shown that certain neuronal populations appear to be vulnerable to both neurofibrillary tangle (NFT) formation and neuronal loss (Terry et al., 1981; Arriagada et al., 1992; Lewis et al., 2000; Zehr et al., 2004; Andorfer et al., 2005; Spires et al., 2006) and that neuronal loss outstrips tangle number by orders of magnitude (Gomez-Isla et al., 1997; Spires et al., 2006). These observations give rise to two possible scenarios: vulnerable neurons develop a tangle which eventually causes the cell to die, then the tangle is cleared; or vulnerable neurons either die or develop a tangle. If the latter is

\footnotetext{
Received July 6, 2007; revised 0ct. 18, 2007; accepted Nov. 16, 2007.

This work was supported by National Institutes of Health Grants AG26249, AG08487, AG00277, EB00768, R01AG26252, and R01-NS46355, the Alzheimer Association Pioneer Award, and John D. French Alzheimer's Disease Foundation Fellowship. A.d.C. is a student in the B2M program at the University of Paris 6, and some of the results in this manuscript will be presented in her thesis. We also thank the Alzheimer's Disease Research Center, Matthew Frosch, Karlotta Fitch, Hisatomo Kowa, and Charles Vanderburg for their contributions.

Correspondence should be addressed to Bradley T. Hyman, MassGeneral Institute for Neurodegenerative Disease, 114 16th Street, Charlestown, MA 02129. E-mail: bhyman@partners.org.

D0I:10.1523/JNEUROSCI.3072-08.2008

Copyright $\odot 2008$ Society for Neuroscience $\quad$ 0270-6474/08/280862-06\$15.00/0
}

the case, tangles could be viewed, paradoxically, as protective or neutral.

Evidence for both possibilities exist although the idea that tangles are toxic has been regarded as most likely. In histologic studies, NFTs are associated with immunohistochemical evidence of caspase activation and caspase-cleavage products, leading to the hypothesis that NFTs cause apoptotic death (Gamblin et al., 2003; Rissman et al., 2004). However, some data from cell culture experiments, mouse models, and disease caused by tau mutations that causes cell death but not tangle formation indicate that protein inclusions may be unnecessary for neuronal death or even protect cells from toxic soluble moieties (Saudou et al., 1998; Reed et al., 2001; Bodner et al., 2006; Spires et al., 2006).

Determining the relationship between NFTs and neuronal death is critical for development of appropriate therapeutic strategies, but analysis has been hampered by limitations of crosssectional studies to discern the fate of tangle-bearing or nontangle-bearing neurons. We therefore developed a method to image NFTs and caspase activation in the intact brain. We find that a small minority of neurons are caspase positive and that the vast majority of caspase positive neurons contain a tangle. If 


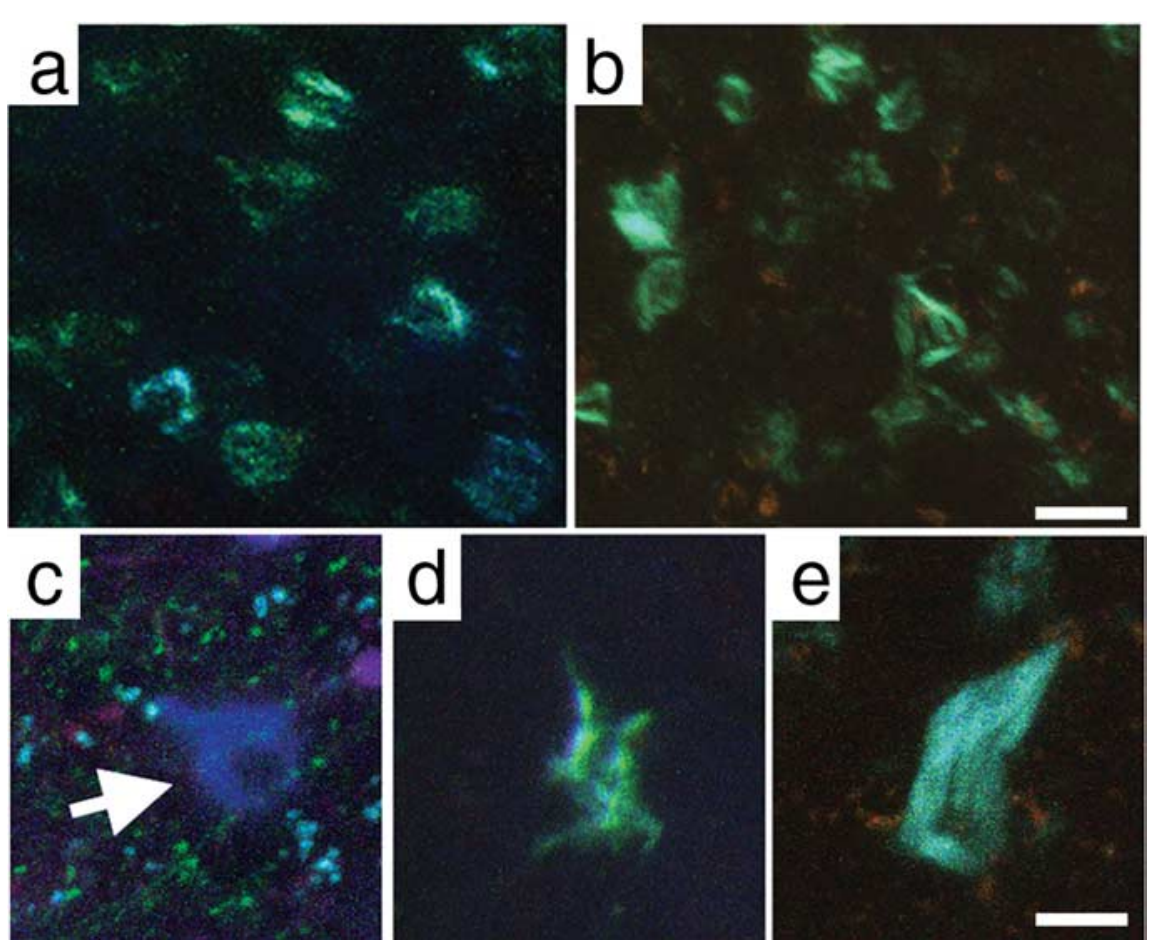

Figure 1. ThioS labels neurofibrillary tangles in vivo. Topical application of $0.025 \%$ ThioS to the cortex of $r$ Tg 4510 mice was used to observe neurofibrillary tangles. $\boldsymbol{a}, \boldsymbol{b}$, Low-magnification micrographs show that NFTs are prevalent throughout the cortex of 6-month-old ( $\boldsymbol{a}$ ) and 9-month-old $(\boldsymbol{b})$ mice. $\boldsymbol{c}-\boldsymbol{e}$, At high resolution, most of these lesions appear blue-green and fibrillar although occasionally less fibrillar lesions are observed which are more blue in color (arrow). ThioS positive neurons were not observed in control animals (data not shown). Scale bars: $\boldsymbol{a}, \boldsymbol{b}, 20 \mu \mathrm{m} ; \boldsymbol{c}-\boldsymbol{e}, 10 \mu \mathrm{m}$.

caspase activation led to acute apoptotic cell death, we would expect rapid degeneration of caspase containing neurons. Instead, we observed that neuronal cell bodies with activated caspases do not degenerate over the course of hours in vivo, and markers for the end stages of apoptosis were absent. Furthermore, caspase activity continued after suppressing the tau transgene, despite cessation of neuronal death, again demonstrating dissociation between caspase activation and acute neuronal death.

\section{Materials and Methods}

Surgery and imaging. Animal experiments were performed under national and institutional guidelines. We used $r$ Tg4510 mice aged 5-10 months $(n=28)$, which reversibly express P301L mutant human tau (SantaCruz et al., 2005). Age-matched littermates expressing only the activator transgene were used as negative controls $(n=20)$. A craniotomy was performed as described previously (Skoch et al., 2004). Before a glass coverslip was sealed over the craniotomy, dura were resected from the cortical surface and a PBS solution containing Thioflavin S $(0.01$, $0.025,0.1$, or $0.2 \%$ ThioS; Sigma, St. Louis, MO) and a fluorescent indicator of caspase activation (FLICA indicators, five times concentration, polycaspase indicator, caspase 3 plus 7 indicator, or caspase 8 indicator; Invitrogen, Eugene OR) was applied for $20 \mathrm{~min}$. Two animals were treated with topical application of z-vad-fmk pan-caspase inhibitor (Promega, Madison, WI; $50 \mu \mathrm{M}$ in PBS) for 30 min before application of polycaspase indicator and ThioS. After initial imaging, the caspase inhibitor was washed out and the caspase indicator and ThioS solution reapplied. A subset of animals ( $n=3 \mathrm{Tg} 4510$ mice, 1 control) were treated with doxycycline (200 ppm in chow; Harlan Tekland, Madison, WI) to suppress tau transgene expression for 2 weeks before surgery.

In vivo imaging was performed using a Bio-Rad (Hercules, CA) 1024ES multiphoton microscope (Bio-Rad) with $800 \mathrm{~nm}$ excitation (Ti: Sapphire laser; Maitai, Spectra-Physics, Fremont, CA) as described previously (Spires et al., 2005). $Z$-stacks of images were collected in $0.5-5$ $\mu \mathrm{m}$ steps with resolutions ranging from $0.1 \mu \mathrm{m} /$ pixel to $0.4 \mu \mathrm{m} /$ pixel.
Animals were killed with an overdose of avertin $(400 \mathrm{mg} / \mathrm{kg})$ and the brain stored in $4 \%$ paraformaldehyde containing $15 \%$ glycerol.

Quantitative PCR. To assess caspase 3, 7, 8, and 9 mRNA levels, quantitative, real-time PCR (qPCR) was performed as described previously (Matsui et al., 2006). Primer sequences are shown in supplemental Table 1 (available at www.jneurosci.org as supplemental material). Transcripts of interest were quantified using a modification of the $\Delta \mathrm{CT}$ method (Fink et al., 1998).

Immunostaining. Immunostaining of tau cleaved at Asp 421/422 [TauC3 antibody described by Gamblin et al. (2003)] was performed on $50 \mu \mathrm{m}$ floating (primary antibody, 1:100; Biosource, Camarillo, CA; secondary anti-mouse Alexa-568, 1:200; Invitrogen).

Statistics. Pearson's correlations and Fisher's $r$ to $z$ ( $p$ values) comparing caspase eight levels and neuron number (Spires et al., 2006) were calculated using StatView (SAS Institute, Cary, NC). To compare transgenic to control levels of caspase eight normalized to glyceraldehyde-3phosphate dehydrogenase (GAPDH) levels, Mann-Whitney $U$ tests were used (caspase mRNA data were not normally distributed). Caspase 8 levels normalized to GAPDH at each age were compared with average control levels, which do not change with age.

\section{Results}

Neurofibrillary pathology is associated with caspase activation in vivo

We used the $r T g 4510$ mouse model (SantaCruz et al., 2005), which reversibly expresses P301L mutant human tau, to investigate neurofibrillary pathology and neurodegeneration in vivo. Thioflavin S (ThioS), a standard histological dye that is fluorescent only when bound to $\beta$ pleated sheet structures, was topically applied to the cortical surface. ThioS labels neurofibrillary pathology in the living mouse brain at 0.025\% concentration in PBS (Fig. 1). In control animals, no ThioS-positive neurons were observed.

rTg4510 mice undergo substantial neuronal loss in the cortex, $50 \%$ loss by 8.5 months of age, assessed previously using stereological counting methods in fixed sections (Spires et al., 2006). Using these stereological data, we estimate that $>1000$ neurons should die each day in the cortical volume of $3.9 \times 10^{9} \mu \mathrm{m}^{3}$ monitored during in vivo imaging. To investigate cell death processes in vivo, we labeled activated caspases with a red fluorescent indicator of polycaspase activation. In $r T g 4510$ cortex, this indicator labeled occasional neurons' soma, nuclei, and proximal dendrites, whereas in other neurons, only the nuclei were labeled (Fig. 2). In $r T g 4510$ cortex, both neurons with and without ThioS-positive inclusions had activated caspases; however, the vast majority of caspase positive neurons contained NFTs. Six percent of observed tangle-containing cells are caspase positive and an estimated $0.1 \%$ of tangle-free neurons are caspase positive. This is a 65 -fold difference, indicating that cells with NFTs are much more likely to be caspase positive than those without.

We also used two other indicators of activated caspases that are more specific than the polycaspase indicator described above. One of these recognizes only activated caspase 8 (an "initiator" caspase) and the other activated caspases 3 and 7 (downstream "executioner" caspases). We observed both caspase 3 plus 7 ( $n=$ 2 animals) and caspase 8 activation $(n=2$ animals, Fig. 2$)$. When 

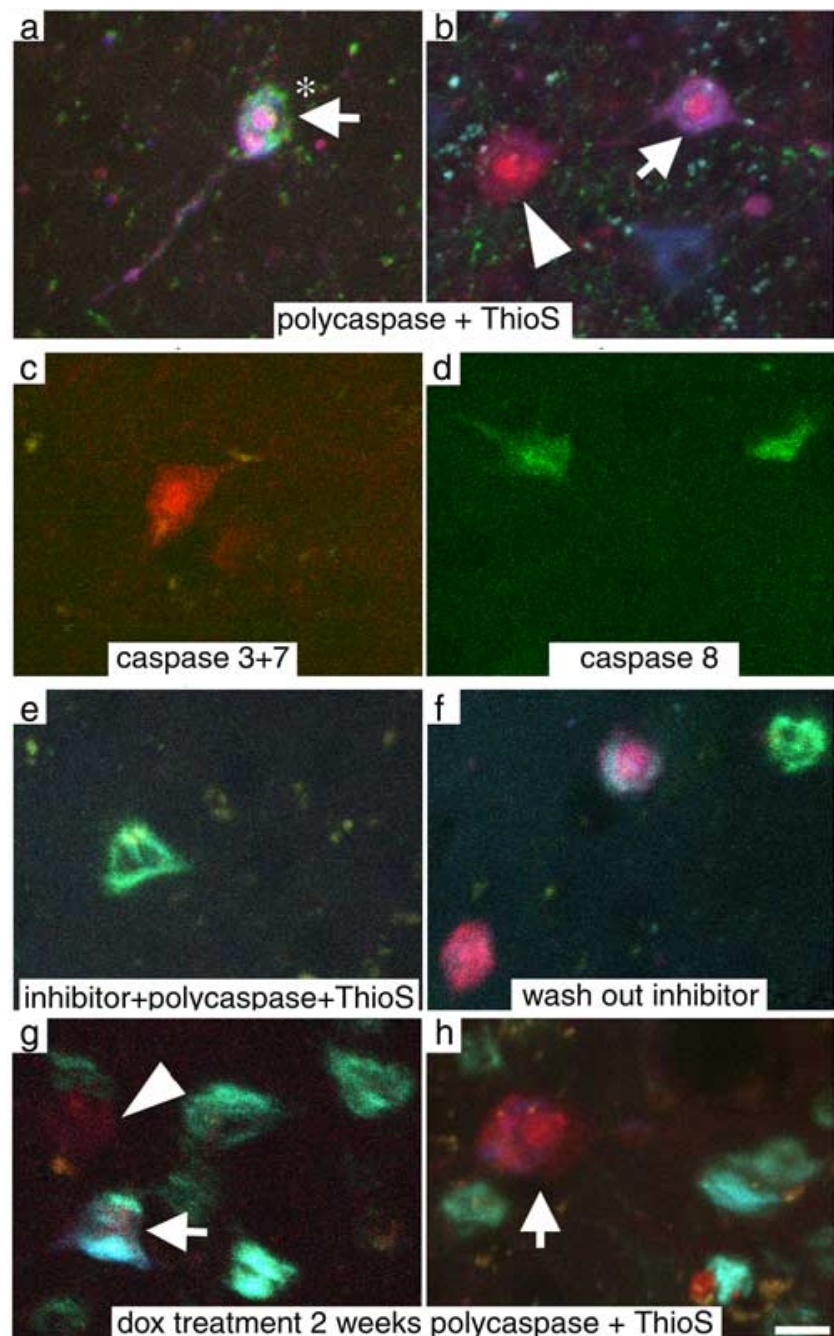

Figure 2. Caspase activation occurs in $r$ Tg 4510 cortex. $\boldsymbol{a}, \boldsymbol{b}, \boldsymbol{e}-\boldsymbol{h}$, An indicator of polycaspase activation applied in vivo (red) shows that activated caspases are present both in cells with ThioS positive tangles (green-blue; arrows) and without (arrowheads). Rarely, cells with active caspases exhibit nuclear fragmentation (asterisks), a morphological characteristic of apoptotic neurons. $\boldsymbol{c}, \boldsymbol{d}$, Indicators specific to caspase 3 and 7 activation (red; $\boldsymbol{c}$ ) and to caspase 8 (green; $\boldsymbol{d}$ ) label neurons showing that both initiator and executioner caspases are active in these mice. $\boldsymbol{e}$, Pretreatment with the pan-caspase inhibitor z-vad-fmk prevented caspase activation although ThioS positive cells are still visible. $\boldsymbol{f}$, After washing out the inhibitor, caspase activation reappears, usually in tangle-bearing cells as expected. $\boldsymbol{g}, \boldsymbol{h}$, Transgene suppression for 2 weeks does not prevent caspase activation. Scale bar, $10 \mu \mathrm{m}$.

used together, the caspase 8 indicator and poly-caspase indicator colocalized as expected, predominantly in cells with NFTs (data not shown). Thus both activator and downstream executioner caspases cascades are activated in a subset of cortical neurons in the $r$ Tg4510 brain.

We considered the possibility that the caspase indicator has some nonspecific or background activation that leads to false positive results. However, caspase-positive neurons were never observed in control animals. As an additional control, two animals were treated with topical z-vad-fmk $(50 \mu \mathrm{M})$, a pan-caspase inhibitor. Pretreatment with caspase inhibitor prevented detection of caspase activation although ThioS positive cells were still visible. After washing out the inhibitor and reapplying caspase indicator, caspase activation appeared in the appropriate proportion of NFT-bearing neurons (Fig. 2).

Based on the in vivo evidence of caspase activation, we sought

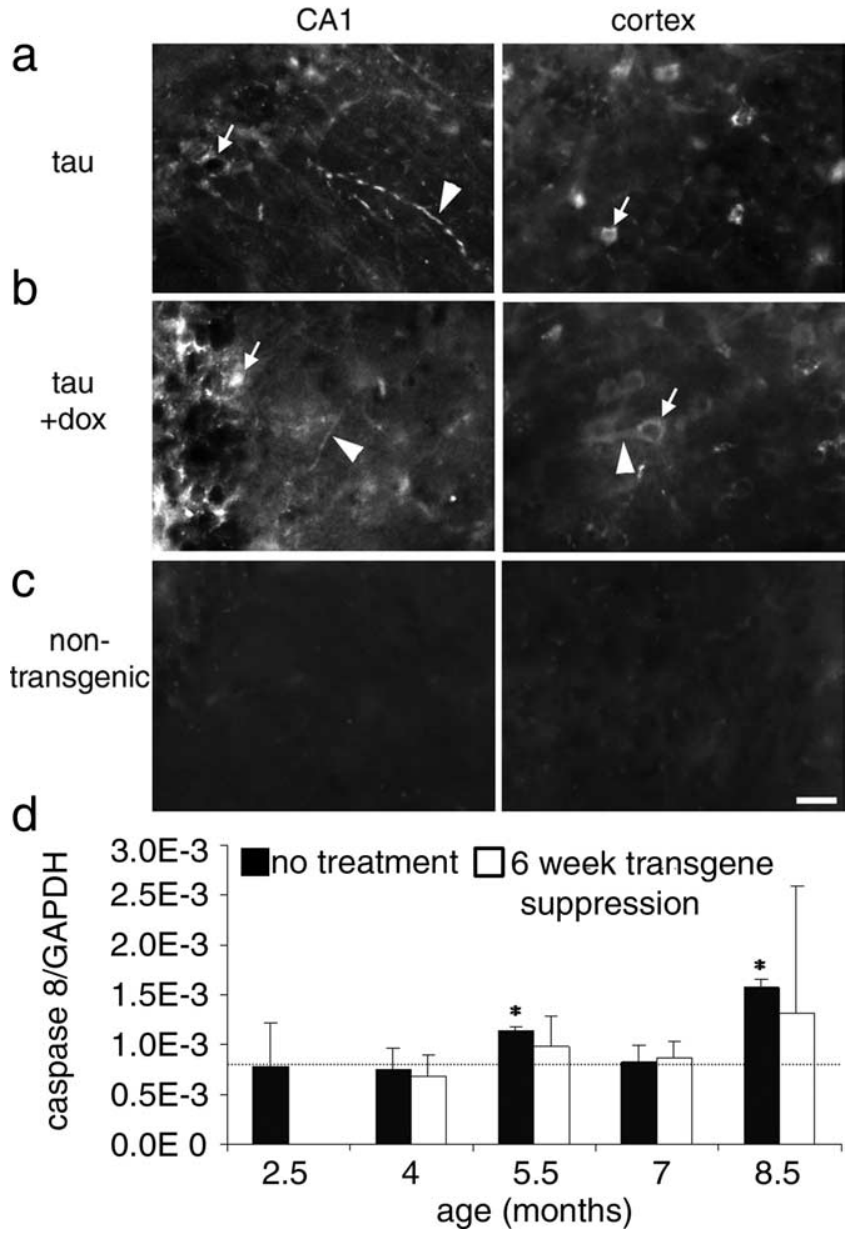

Figure 3. Histological and molecular evidence of caspase upregulation. $\boldsymbol{a}$, Immunostaining for tau cleaved at Aps 421/422 revealed cell bodies (arrows) and processes (arrowheads) in the hippocampus and cortex of $r \mathrm{Tg} 4510$ tau transgenic mice. $\boldsymbol{b}$, This activation persisted after transgene suppression with doxycycline (dox). c, No staining was observed in nontransgenic control mice. $\boldsymbol{d}$, Caspase 8 mRNA levels (normalized to GAPDH) are elevated in $r \mathrm{Tg} 4510$ mice at 5.5 and 8.5 months compared with control levels (dotted line). Transgene suppression does not significantly alter caspase levels. ${ }^{*} p<0.05$, Mann-Whitney $U$ test. Scale bar, $20 \mu \mathrm{m}$.

additional data on the relationship of tau overexpression, NFTs, and caspase activation. Immunostaining for a tau epitope that occurs after caspase cleavage at Asp421/422 revealed positive cell bodies and neuronal processes in the hippocampus and cortex of rTg4510 mice, but not in nontransgenic animals (Fig. 3). Both dendrites and axons were clearly labeled in the CA1 region of the hippocampus. Cortical neurons were only rarely positive for caspase-cleaved tau, in agreement with the rarity of caspase positive cells observed in vivo. Tau can be cleaved at Asp421/422 by caspases 3, 7, and 8 (Gamblin et al., 2003).

We also evaluated molecular methods of assessing caspase involvement. Previously, we noted that the mRNA for caspases 7 and 8 (but not 3 and 9) were elevated in AD brain (Matsui et al., 2006). We therefore performed qPCR on mRNA extracted from rTg4510 animals at 2.5, 4, 5.5, 7, and 8.5 months (Fig. 3). These results showed elevated caspase $8 \mathrm{mRNA}$ levels at 5.5 and 8.5 months, indicating that the expression of this initiator caspase is upregulated in these mice as well as being activated as shown by the cleaved tau immunostaining and in vivo indicator experiments. 

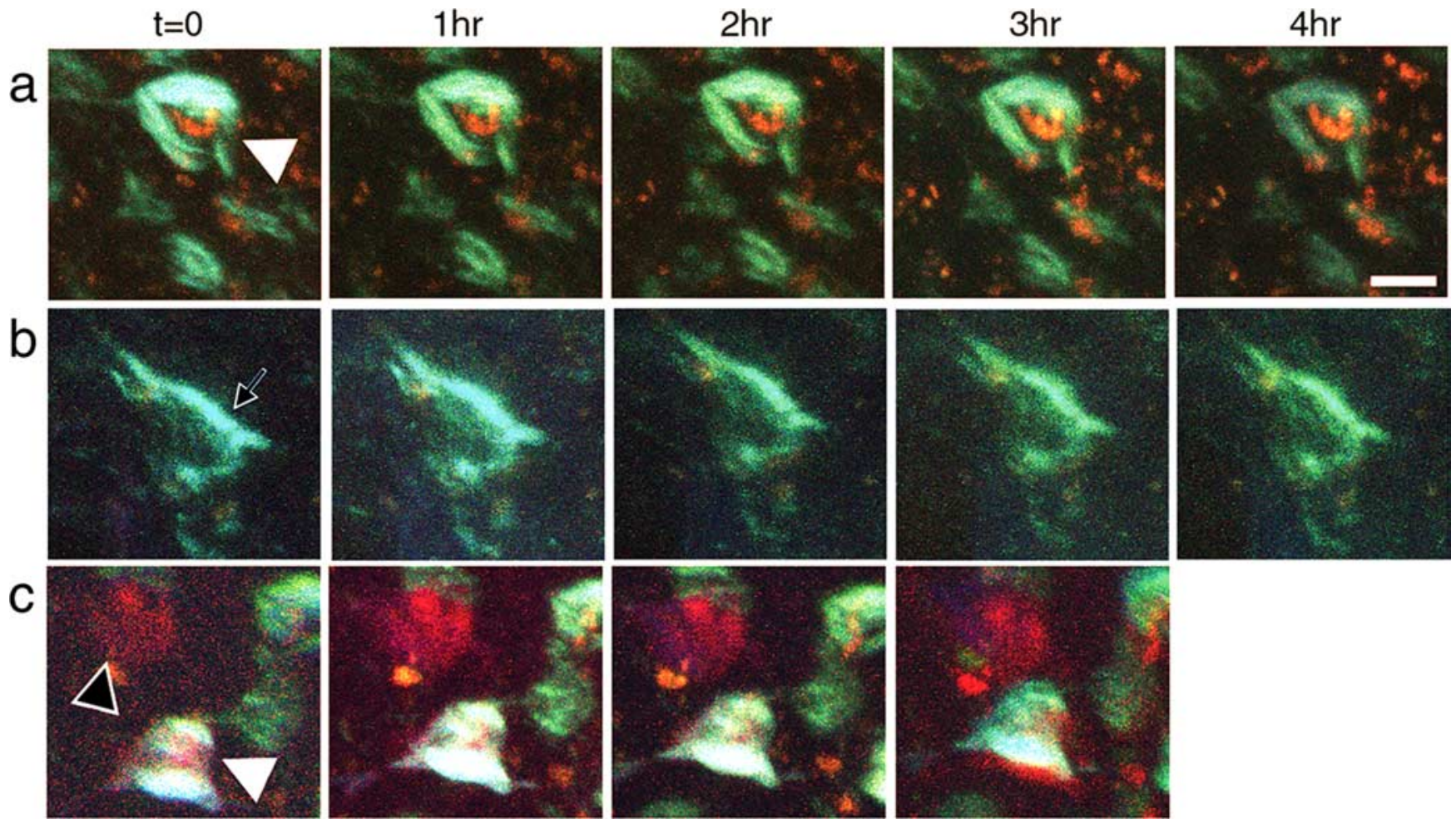

Figure 4. Caspase activation does not lead to rapid degeneration and persists after transgene suppression. $\boldsymbol{a}$, In untreated $r$ Tg4510 animals, caspase positive cell bodies (red) and cells with NFTs (blue-green) remain stable over the course of hours of imaging. $\boldsymbol{b}, \boldsymbol{c}$, Caspase positive cells remain even after transgene suppression and these cell bodies similarly do not degenerate over the course of hours. The black arrowhead indicates a cell with activated caspases but no tangle, white arrowheads show cells with both NFTs and activated caspases, and the black arrow shows a cell with NFTs but no active caspases. Scale bar, $10 \mu \mathrm{m}$.

\section{Caspase activation does not lead to classical apoptotic death of neurons}

These data suggest that caspase activation can be detected in a small number of individual neurons in the cortex, and those are most likely to contain NFTs. Together with our previous observations that having NFTs was not sufficient, in most neurons, to cause neuronal death (Spires et al., 2006), this result was surprising. Moreover, caspase positive neuronal nuclei appeared morphologically identical to their caspase negative neighbors (only two fragmented nuclei of hundreds observed). If apoptosis were occurring, we would expect rapid degeneration of neurons as well as fragmenting nuclei, so we imaged some neurons with NFTs and caspase activation hourly for $4 \mathrm{~h}$ (a total of five image time points). Neurons containing NFTs remained unchanged over these imaging sessions, even if they were caspase positive (Fig. 4).

Suppression of the tau transgene for 6 weeks in this model halts ongoing neuronal death without affecting NFTs (SantaCruz et al., 2005; Spires et al., 2006). We suppressed the tau transgene for 2 weeks by treatment with oral doxycycline. As expected, NFTs persisted for the 2 week interval. Surprisingly, however, caspase activation also remained as prevalent as without transgene suppression (Figs. 2, 4, supplemental Table 2, available at www.jneurosci.org as supplemental material).

Histological and molecular experiments also support the dissociation of caspase activation and neuronal death. Transgene suppression for 2 weeks did not remove caspase-cleaved tau from neurons, nor did 6 weeks of suppression change the levels of caspase 8 mRNA (Fig. 3). Tissue from the same animals used for qPCR experiments was previously used to estimate neuron and tangle numbers in various brain regions (Spires et al., 2006). Comparing neuronal counts from one hemisphere with the
qPCR results from the contralateral hemisphere showed that caspase 8 levels are inversely correlated with neuron number. This indicates an association of caspase 8 upregulation with neuronal loss (correlation, $-0.611 ; p=0.002$ ). However, the lack of change in caspase 8 levels or caspase cleaved tau, and the presence of activated caspases in vivo after transgene suppression argues that caspase production and activation are not sufficient to support ongoing neuronal death, which is halted over the same time period of suppression.

We further investigated whether neurons in rTg4510 cortex die via classical caspase-associated apoptosis using histological markers. Neurons in rTg4510 mice and control nontransgenic animals were negative for cleaved PARP [poly(ADP-ribose) polymerase], terminal deoxynucleotidyl transferase-mediated biotinylated UTP nick end labeling (TUNEL), and cleaved caspase-3 immunostaining (supplemental Fig. 1, available at www.jneurosci.org as supplemental material), indicating there is not apoptosis occurring at least as could be detected with these three markers. Additionally, nuclei of the neurons appeared normal in histological sections [thousands of cresyl violet stained nuclei were examined in a previous stereological study (Spires et al., 2006)]. Furthermore, Western blots probing for fodrin did not show an increase in caspase cleaved fodrin in rTg4510 brain above the low background level seen in control animals (data not shown). Nor was there any evidence of re-entry into the cell cycle or autophagy as assessed by PCNA (proliferating cell nuclear antigen), cdk4, or LAMP2 (lysome-associated membrane protein-2) immunostaining (data not shown). The detection of activated caspases ( 8 and 3 plus 7 ) in vivo, but the absence of cleaved caspase 3-positive neurons detected in histological sections may indicate that the in vivo indicator detects low levels of 
caspase activation (sufficient to generate caspase-cleaved tau in neurons), and that these tangle-bearing neurons mount an effective antiapoptotic strategy that prevents progression to neuronal death.

\section{Discussion}

To investigate the role of neurofibrillary tangles in tau-associated neuronal death, we have developed a method, using multiphoton microscopy, to directly observe neurofibrillary lesions and caspase activation in vivo. This is the first time that NFTs or caspase activation has been observed in the living brain. These novel techniques demonstrate an unexpected set of relationships: caspase activation can be detected in a small subset of neurons, the vast majority of which contain tangles, but surprisingly, this activation is not a harbinger of inevitable neuronal death. Neurons that are caspase positive remain so, morphologically normal, for up to $4 \mathrm{~h}$ of continuous imaging. Even more strikingly, neurons that contain NFTs remain caspase positive 2 weeks after transgene suppression, which is unexpected because suppression for 6 weeks prevents additional neuronal loss. The absence of TUNEL staining and other evidence of apoptotic sequelae also argue against classical apoptosis as the mechanism of neuronal death in this model. Our results are surprising because whereas activation of initiator caspases has been reported as a chronic event preceding neuronal death in a model of familial amyotrophic lateral sclerosis (Pasinelli et al., 2000), executioner caspase activation is viewed as a direct and inevitable path toward apoptotic cell death that occurs within hours (Chang et al., 2002; Young et al., 2005). Our results indicate that initiator and executioner caspases appear to be activated in tangle-bearing neurons, but longitudinal in vivo imaging uncovers a dissociation between caspase activation and acute neuronal death.

These data address the important and debated issue of whether apoptosis is the mechanism of neuronal death in tauopathies. Evidence for apoptosis in tauopathies is controversial. Studies in human AD tissue indicate that caspases are upregulated and activated caspases and their cleavage products are associated with NFTs (Rohn et al., 2001; Gamblin et al., 2003; Matsui et al., 2006), leading to the hypothesis that tangles contribute to apoptotic neuronal death by activating caspases. However, these experiments from postmortem tissue do not allow direct confirmation that neurons with activated caspases actually undergo apoptosis. In several other mouse models of tauopathy, apoptosis was not found to be a major contributor to neuronal death (Allen et al., 2002; Zehr et al., 2004; Andorfer et al., 2005). Our data show that caspases are activated and associated with NFTs, but caspase activation is not sufficient to cause acute neuronal degeneration arguing against apoptosis as the primary mechanism for cell death in this model.

We propose that tangle-bearing neurons mount an antiapoptotic strategy that delays neuronal death despite caspase activation. The antiapoptotic activity could consist of sequestering active caspases into cell compartments where they are not damaging or upregulation of proteins that inhibit apoptosis (Cotman et al., 2005). The absence of cleaved caspase immunostaining in our model may reflect a low level of activity that is detectable by the in vivo indicator, and sufficient to cause tau cleavage. The latter cleavage product would be expected to accumulate in NFTs, which may allow for its detection; it is possible that caspase cleaved tau even contributes to NFT formation or stabilization (Gamblin et al. 2003). In cell culture and animal models, tau phosphorylation can protect cells from apoptosis ( $\mathrm{Li}$ et al., 2007). The delayed (nonapoptotic) neuronal death in the
rTg4510 model may be similar to that observed in cultured neurons when delayed caspase-independent death is observed in cells treated with an apoptosis-inducing agent while caspases are inhibited (Lang-Rollin et al., 2003).

In vivo imaging of NFTs allows us to also address the relationship between NFTs and neurodegeneration in tauopathies. There are compelling data supporting the toxicity of aggregated tau in vitro (Khlistunova et al., 2006); however, data from cell models of Huntington- and Parkinson-related inclusions (Saudou et al., 1998; Bodner et al., 2006), and from the observation that expression of mutant tau in human tauopathies (Reed et al., 2001) and in Drosophila (Wittmann et al., 2001) can lead to neuronal loss without passing through a tangle stage raise the possibility that protein inclusions may be unnecessary for neuronal death or even be protective. Our previous work on tissue from $r \mathrm{Tg} 4510$ mice showed that substantial neuronal loss occurs in dentate gyrus, which develops very few NFTs, and conversely that there is no cell loss in striatum despite robust amounts of tangle formation, raising the possibility that NFTs are not required and are not sufficient for cell death in this model (Spires et al., 2006). We now show that NFTs are associated with at least low levels of caspase activation, and thus are likely associated with neurotoxicity.

However, NFT-bearing cells apparently can mount an antiapoptotic strategy that slows down or abrogates apoptosis (Cotman et al., 2005), even in the presence of executioner caspases because activation can occur without obligatory acute neuronal death. Thus, although NFTs may be associated with toxic phenomenon, they do not appear to be coupled with acute neurodegeneration in this tauopathy model.

\section{References}

Allen B, Ingram E, Takao M, Smith MJ, Jakes R, Virdee K, Yoshida H, Holzer M, Craxton M, Emson PC, Atzori C, Migheli A, Crowther RA, Ghetti B, Spillantini MG, Goedert M (2002) Abundant tau filaments and nonapoptotic neurodegeneration in transgenic mice expressing human P301S tau protein. J Neurosci 22:9340-9351.

Andorfer C, Acker CM, Kress Y, Hof PR, Duff K, Davies P (2005) Cell-cycle reentry and cell death in transgenic mice expressing nonmutant human tau isoforms. J Neurosci 25:5446-5454.

Arriagada P, Growdon J, Hedley-Whyte E, Hyman B (1992) Neurofibrillary tangles but not senile plaques parallel duration and severity of Alzheimer's disease. Neurology 42:631-639.

Bodner RA, Outeiro TF, Altmann S, Maxwell MM, Cho SH, Hyman BT, McLean PJ, Young AB, Housman DE, Kazantsev AG (2006) From the cover: pharmacological promotion of inclusion formation: a therapeutic approach for Huntington's and Parkinson's diseases. Proc Natl Acad Sci USA 103:4246-4251.

Chang LK, Putcha GV, Deshmukh M, Johnson Jr EM (2002) Mitochondrial involvement in the point of no return in neuronal apoptosis. Biochimie 84:223-231.

Cotman CW, Poon WW, Rissman RA, Blurton-Jones M (2005) The role of caspase cleavage of tau in Alzheimer disease neuropathology. J Neuropathol Exp Neurol 64:104-112.

Fink L, Seeger W, Ermert L, Hanze J, Stahl U, Grimminger F, Kummer W, Bohle RM (1998) Real-time quantitative RT-PCR after laser-assisted cell picking. Nat Med 4:1329-1333.

Gamblin TC, Chen F, Zambrano A, Abraha A, Lagalwar S, Guillozet AL, Lu M, Fu Y, Garcia-Sierra F, LaPointe N, Miller R, Berry RW, Binder LI, Cryns VL (2003) Caspase cleavage of tau: linking amyloid and neurofibrillary tangles in Alzheimer's disease. Proc Natl Acad Sci USA 100:10032-10037.

Gomez-Isla T, Hollister R, West H, Mui S, Growdon JH, Petersen RC, Parisi JE, Hyman BT (1997) Neuronal loss correlates with but exceeds neurofibrillary tangles in Alzheimer's disease. Ann Neurol 41:17-24.

Khlistunova I, Biernat J, Wang Y, Pickhardt M, von Bergen M, Gazova Z, Mandelkow E, Mandelkow EM (2006) Inducible expression of Tau repeat domain in cell models of tauopathy: aggregation is toxic to cells but can be reversed by inhibitor drugs. J Biol Chem 281:1205-1214. 
Lang-Rollin ICJ, Rideout HJ, Noticewala M, Stefanis L (2003) Mechanisms of caspase-independent neuronal death: energy depletion and free radical generation. J Neurosci 23:11015-11025.

Lewis J, McGowan E, Rockwood J, Melrose H, Nacharaju P, Van Slegtenhorst M, Gwinn-Hardy K, Paul Murphy M, Baker M, Yu X, Duff K, Hardy J, Corral A, Lin WL, Yen SH, Dickson DW, Davies P, Hutton M (2000) Neurofibrillary tangles, amyotrophy and progressive motor disturbance in mice expressing mutant (P301L) tau protein. Nat Genet 25:402-405.

Li HL, Wang HH, Liu SJ, Deng YQ, Zhang YJ, Tian Q, Wang XC, Chen XQ, Yang Y, Zhang JY, Wang Q, Xu H, Liao FF, Wang JZ (2007) Phosphorylation of tau antagonizes apoptosis by stabilizing beta-catenin, a mechanism involved in Alzheimer's neurodegeneration. Proc Natl Acad Sci USA 104:3591-3596.

Matsui T, Ramasamy K, Ingelsson M, Fukumoto H, Conrad C, Frosch MP, Irizarry MC, Yuan J, Hyman BT (2006) Coordinated expression of caspase 8, 3 and 7 mRNA in temporal cortex of Alzheimer disease: relationship to formic acid extractable abeta42 levels. J Neuropathol Exp Neurol 65:508-515.

Pasinelli P, Houseweart MK, Brown Jr RH, Cleveland DW (2000) Caspase-1 and -3 are sequentially activated in motor neuron death in $\mathrm{Cu}, \mathrm{Zn}$ superoxide dismutase-mediated familial amyotrophic lateral sclerosis. Proc Natl Acad Sci USA 97:13901-13906.

Reed LA, Wszolek ZK, Hutton M (2001) Phenotypic correlations in FTDP17. Neurobiol Aging 22:89-107.

Rissman RA, Poon WW, Blurton-Jones M, Oddo S, Torp R, Vitek MP, LaFerla FM, Rohn TT, Cotman CW (2004) Caspase-cleavage of tau is an early event in Alzheimer disease tangle pathology. J Clin Invest 114:121-130.

Rohn TT, Head E, Su JH, Anderson AJ, Bahr BA, Cotman CW, Cribbs DH (2001) Correlation between caspase activation and neurofibrillary tangle formation in Alzheimer's disease. Am J Pathol 158:189-198.

SantaCruz K, Lewis J, Spires T, Paulson J, Kotilinek L, Ingelsson M, Guima- raes A, DeTure M, Ramsden M, McGowan E, Forster C, Yue M, Orne J, Janus C, Mariash A, Kuskowski M, Hyman B, Hutton M, Ashe KH (2005) Tau suppression in a neurodegenerative mouse model improves memory function. Science 309:476-481.

Saudou F, Finkbeiner S, Devys D, Greenberg ME (1998) Huntingtin acts in the nucleus to induce apoptosis but death does not correlate with the formation of intranuclear inclusions. Cell 95:55-66.

Skoch J, Hickey GA, Kajdasz ST, Hyman BT, Bacskai BJ (2004) In vivo imaging of amyloid-beta deposits in mouse brain with multiphoton microscopy. In: Amyloid proteins: methods and protocols (Sigurdsson EM, ed), pp 349-364. Totowa, NJ: Humana.

Spires TL, Meyer-Luehmann M, Stern EA, McLean PJ, Skoch J, Nguyen PT, Bacskai BJ, Hyman BT (2005) Dendritic spine abnormalities in amyloid precursor protein transgenic mice demonstrated by gene transfer and intravital multiphoton microscopy. J Neurosci 25:7278-7287.

Spires TL, Orne JD, SantaCruz K, Pitstick R, Carlson GA, Ashe KH, Hyman BT (2006) Region-specific dissociation of neuronal loss and neurofibrillary pathology in a mouse model of tauopathy. Am J Pathol 168:1598-1607.

Terry RD, Peck A, DeTeresa R, Schechter R, Horoupian DS (1981) Some morphometric aspects of the brain in senile dementia of the Alzheimer type. Ann Neurol 10:184-192.

Wittmann CW, Wszolek MF, Shulman JM, Salvaterra PM, Lewis J, Hutton M, Feany MB (2001) Tauopathy in Drosophila: neurodegeneration without neurofibrillary tangles. Science 293:711-714.

Young C, Roth KA, Klocke BJ, West T, Holtzman DM, Labruyere J, Qin YQ, Dikranian K, Olney JW (2005) Role of caspase-3 in ethanol-induced developmental neurodegeneration. Neurobiol Dis 20:608-614.

Zehr C, Lewis J, McGowan E, Crook J, Lin WL, Godwin K, Knight J, Dickson DW, Hutton M (2004) Apoptosis in oligodendrocytes is associated with axonal degeneration in P301L tau mice. Neurobiol Dis 15:553-562. 